\title{
Secured Wireless Communication to Connected Vehicles
}

\author{
D. Thirupathi Rao ${ }^{1}$, Dr. M. Sampath Kumar ${ }^{2}$ \\ ${ }^{1}$ M.Tech Schlor, Department of Computer Science and Systems Engineering, Andhra University, Vishakapatnam \\ ${ }^{2}$ Professor, Department of Computer Science and Systems Engineering, Andhra University, Vishakapatnam
}

\begin{abstract}
To design a model with principle of operation according to natural aspects which are related to real time applications, which are creating problems like accidents and thefting in daily life. Hence, proposed a system called Autonomous car with Intelligence system. A prototype model is designed and constructed. Secured wireless communication is used to drive the vehicle by using AI techniques.
\end{abstract}

Keywords: ATmega328, L293D chip, Servo motor, Ultrasonic Sensor

\section{Introduction}

Traffic accidents are a major source of disability and mortality worldwide. Every year, 1.2 million people die and up to 50 million people are injured. Autonomous or highly aware cars have the potential to reduce these numbers dramatically. However, building a self-driving car that exceeds human driving performance is not easy. While traffic injuries and deaths are large in number, they are relatively infrequent. In the United States, a human driver makes a fatal mistake only once in approximately 88 million miles. In daily life we see many problems which are playing major role in real time environment like accidents which is creating destruction of cars and loosing life of others so this paper understands you about Securing Wireless Communications of Connected Vehicles with self-driving which is also known as Driverless cars. These cars have many features like, car detection, lane keeping, parking, and intersections were included. Important exclusions were high-speed (highway) driving, traffic lights, bicycles, pedestrians, and the ability to drive without a highly accurate GPS course skeleton.

\section{Hardware Design}

\subsection{List of Hardware}

A pre-built four-wheel drive (4WD) is used as a base on which following hardware components are fit:

- Atmega for GPU and CPU computations

- Blue tooth to connect car to app

- Motor driver IC L293D which can control four motors

- $12 \mathrm{~V}$ batteries to provide power

- Static IP-camera

- Ultrasonic sensor to detect obstacles

- Buzzer is used to alert the vehicle behind the car when it is stopped.

\subsection{Hardware and Software Description}

\subsubsection{Arduino-ATmega 328 Microcontroller}

The Arduino Uno is an open-source microcontroller board based on the ATmega328. It has 14 digital input/output pins (of which 6 can be used as PWM outputs) and 6 analog input. It contains everything needed to support the microcontroller, and it can be simply connected to a computer with a Universal Serial Bus (USB) cable to get started.

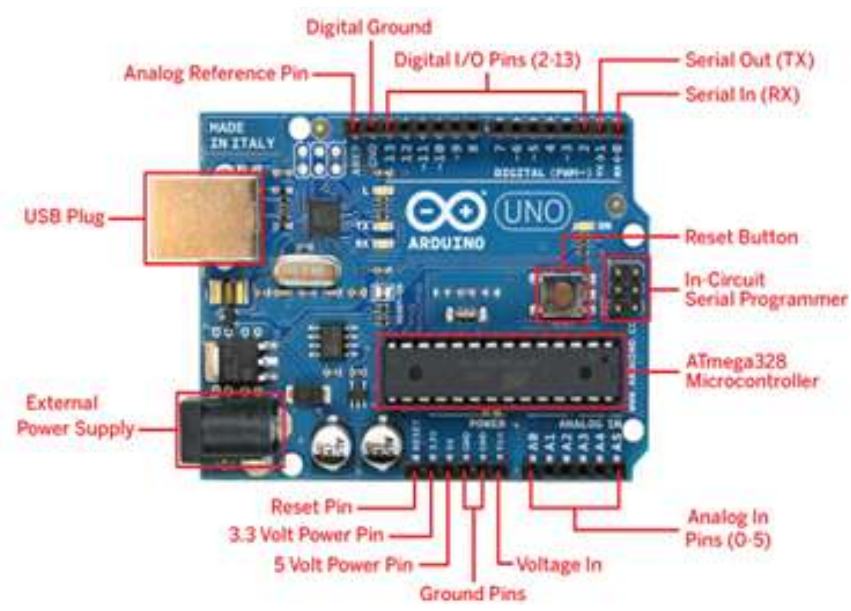

Figure 1: Arduino control board

The Arduino Uno can be programmed with the Arduino Integrated Development Environment (IDE). The C-based simple program code for the Arduino is referred to as a sketch. Collection of sketches for specific functionalities is referred to as libraries. The Arduino can be programmed up to $32 \mathrm{~KB}$ memory. Arduino can function autonomously without being connected to a computer, or alternatively programmed to respond mainly to commands sent from the computer via various software interfaces or to the data acquired from the input channels. The Arduino UNO based on ATMega-328 Microcontroller Aurdino micro controller:

\subsubsection{IP Camera}

An Internet protocol camera, or IP camera, is a type of digital video camera commonly employed for surveillance, and which, unlike analog closed circuit television (CCTV) cameras, can send and receive data via a computer network and the Internet. Although most cameras that do this are webcams, the term "IP camera" or "netcam" is usually applied only to those used for surveillance. An IP camera is typically either centralized (requiring a central network video

\section{Volume 6 Issue 12, December 2017}

\author{
www.ijsr.net
}




\section{International Journal of Science and Research (IJSR) \\ ISSN (Online): 2319-7064}

Index Copernicus Value (2015): 78.96 | Impact Factor (2015): 6.391

recorder (NVR) to handle the recording, video and alarm management) or decentralized (no NVR needed, as camera can record to any local or remote storage media).

\subsubsection{L293D H-bridge integrated circuit:}

L293D is a typical Motor driver or Motor Driver IC which allows DC motor to drive on either direction. L293D is a 16pin IC which can control a set of two DC motors simultaneously in any direction. It means that you can control two DC motor with a single L293D IC. In a single L293D chip there are two h-Bridge circuit inside the IC which can rotate two dc motor independently-bridge is a circuit which allows the voltage to be flown in either direction-bridge IC are ideal for driving a DC motor. Due its size it is very much used in robotic application for controlling DC motors.

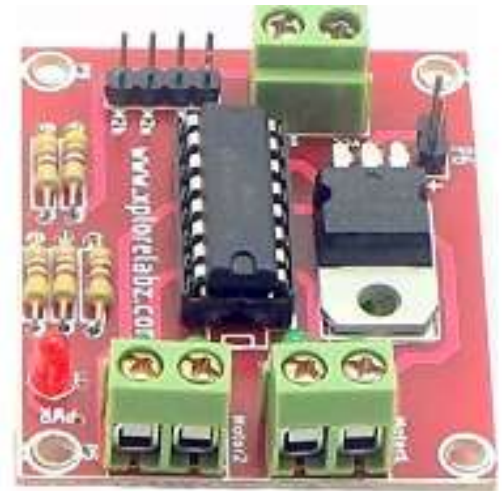

Figure 2: L293D Circuit diagram

\subsubsection{Ultrasonic sensor}

Ultrasonic sensors (also known as transceivers when they both send and receive, but more generally called transducers) evaluate attributes of a target by interpreting the echoes from radio or sound waves respectively [1]. In this project, they are used to detect the distance of obstacles from the car.It detects and plans the algorithm called path detection according to priority with artificial intelligence

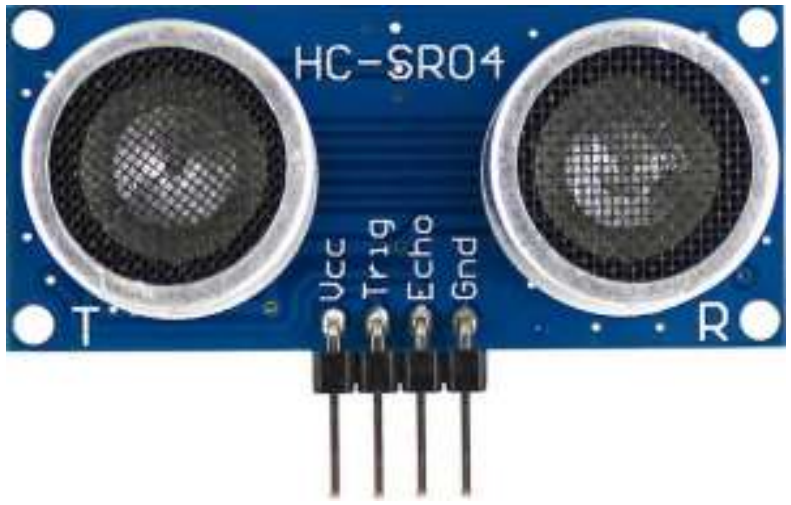

Figure 3: Ultrasonic sensor

\section{Block Diagram and System Implementation}

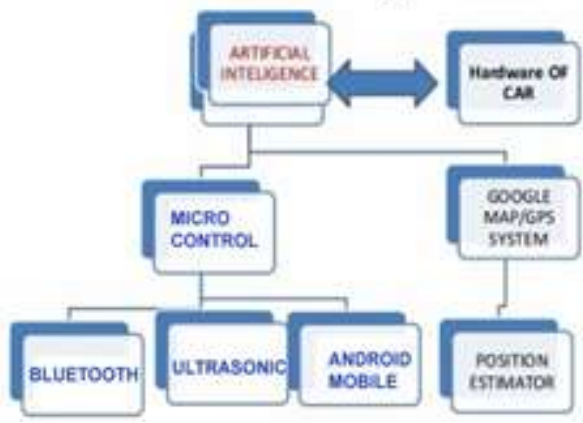

Figure 4:Block Diagram

The system is implemented using the Aurdino microcontroller for the design of a prototype of the proposed model. When the moving is enabled, the steering starts to accelerate at a given speed. The ultrasonic sensors are fitted on the front side. These sensors detects any possible obstacle. The sensors always receive low to high pulse from the receiving end when there is no obstacle. When two lowpulse waves are detected, the space is meant to be free. When the car moves into the free parking space, the ultrasonic sensor probe for possible obstacle in order to park the car in a correct orientation and signals the sensor so that the wheels of the robot rotate accordingly. Once the car is parked and locked, a message is sent to the corresponding user's mobile number via BOT that is embedded into the electronic control unit of the car. This movement detection message is sent to the user about any theft attack. For the retrieval of the driverless car, the mode of control car can be either automatic or manual. This mode is selected by the user from the android application that is available on his/her Smartphone. If the manual mode is selected, the direction of the car movement is selected from the android application. The android application is exclusively developed for this purpose. The corresponding selection is sent to the BOT i.e BLUTOOTH OF THINGS modem of the car driven remotely using the Smartphone to any descried location. The accuracy of the maneuver depends on the condition of the roadway where the car is driven. If the auto mode is selected, the probing is done using the ultrasonic sensors in the usual way of obstacle detection. The car is driven either to the location where it was originally left by the auto park mode or car can be drove to a pre-defined location by tracking through GPS tracking.

\subsection{MIT APP Inventor}

The MIT App Inventor project seeks to democratize software development by empowering all people, especially young people, to move from technology consumption to technology creation. As Android continues to grow beyond smart phones, it will become the brains behind invisible, ubiquitous cloud-connected computing. These days the demand of smart phone is being increased and we have different types of client e.g. Touch Phone, tables, Note etc.

The demands of the applications for these smart clients are constantly increasing and we have several operating systems for these smart clients e.g. Android, IOS and Windows etc 


\section{International Journal of Science and Research (IJSR)}

ISSN (Online): 2319-7064

Index Copernicus Value (2015): 78.96 | Impact Factor (2015): 6.391

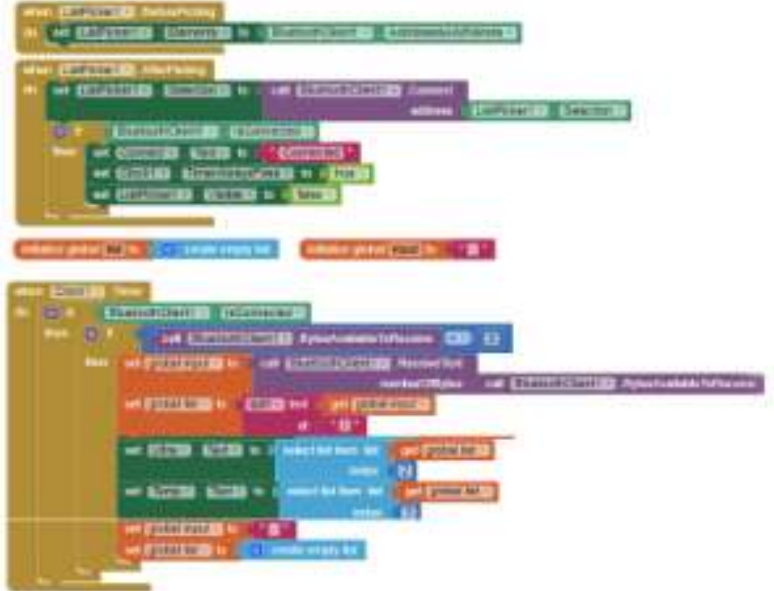

Figure 5: Mit app inventor block editor

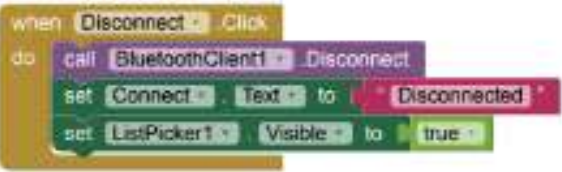

Figure 6: MIT APP Inventor Disconnect Button

\subsection{Front End of MIT APP Inevntor}

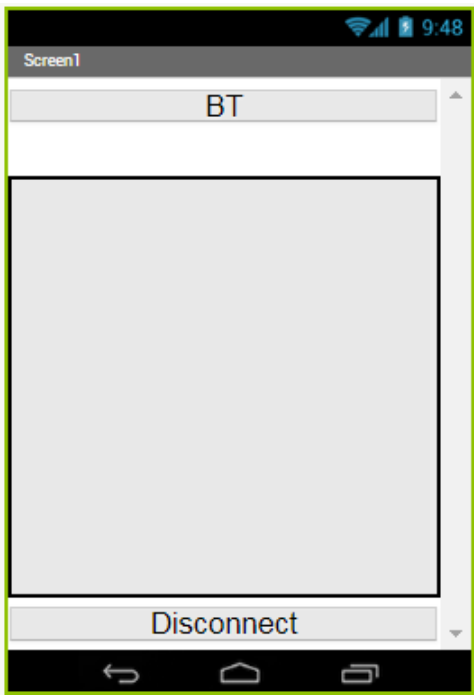

Figure 7: Front end of mit app inventor

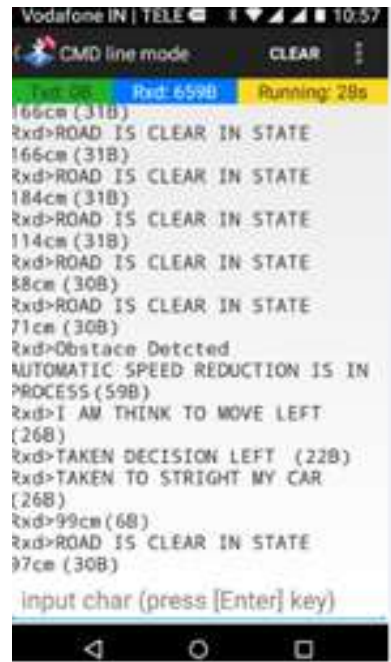

Figure 8: Serial monitor app

\section{Result}

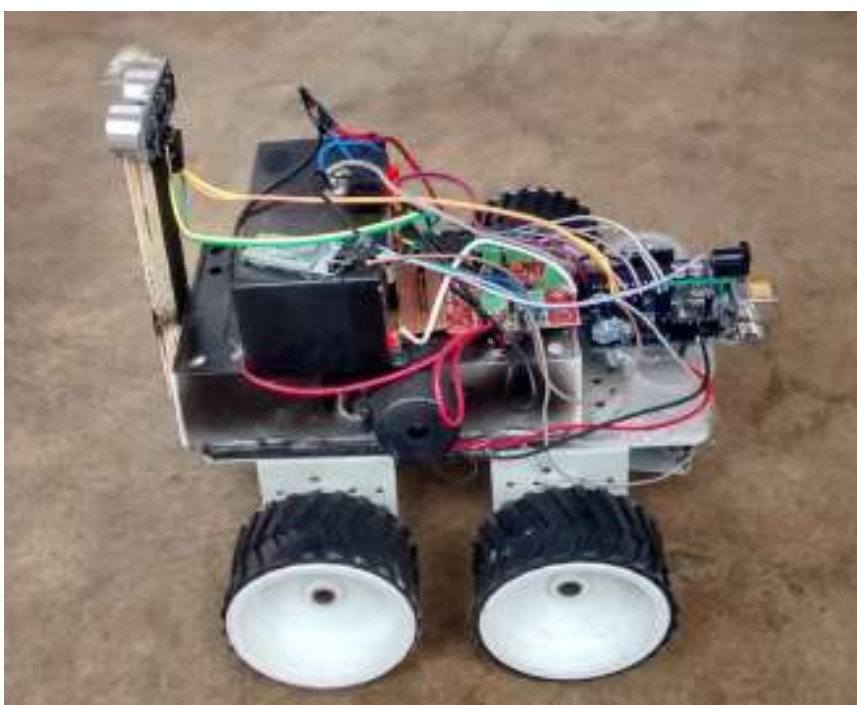

Figure 9: Driverless Car Prototype

\section{Conclusion}

In this paper, a method to make a self-driving car is presented. The different hardware components and their assembly are clearly described. Using ultrasonic sensors, the collisions with obstacles is avoided. When accidents are occurred message is sent to the mobile terminal through wireless network. GPS tracking is done by showing the latitudinal and longitudinal values by google maps. The algorithm mentioned in the paper has been successfully implemented on a small autonomous car.

\section{References}

[1] Prinkle Sharma; Hong Liu; Honggang Wang; Shelley Zhang A. Securing wireless communications of connected vehicles with artificialintelligence 2017 IEEE International Symposium on Technologies for Homeland Security (HST)

[2] Y. J. Abueh and H. Liu, "Message Authentication in Driverless Cars," in 2016 IEEE Symposium on Technologies for Homeland Security (HST), 2016.

[3] B. Vlasic and N. E. Boudette, "Self-Driving Tesla was Involved in Fatal Crash, U.S. Says," Business Day, 30 June 2016

[4] S. Zeadally, R. Hunt, Y. S. Chen, A. Irwin and A. Hassan, "Vehicular Ad Hoc Networks (VANETS): Status, Results and Challenges.," Telecommunication Systems, vol. 50, no. 4, pp. 217-241, August 2012.

[5] L. Needhi, S. Bhushan and M. Mahajan, "Intelligent Hazard Routing for VANETs with Point of Interest Evaluation Technique," International Journal of Computer Science and Mobile Computing, vol. 4, no. 7, pp. 116-121, 2015

[6] M. Booysen, "Simulating VANET and ITS (using SUMO and OMNET++)," Seminar at UniRC, 2012.

[7] H.-C. Hsiao, A. Studer, C. Chen, A. Perrig, F. Bai, B. Bellur and A. Iyer, "Flooding-Resilient Broadcast Authentication for VANETs," in 17th annunal

Volume 6 Issue 12, December 2017

www.ijsr.net

Licensed Under Creative Commons Attribution CC BY 
international conference on Mobile computing networking, New York, 2011

\section{Author Profile}

D. Thirupathi Rao, pursuing his M.Tech in the department of Computer Science and Systems Engineering, Andhra University College of Engineering, Visakhapatnam, A.P., India. He obtained his B.Tech(CSE) from ANU Guntur.

Dr. M. Sampath Kumar, M.E., Ph.D., working as Professor in the department of Computer Science and Systems Engineering, Andhra University, Visakhapatnam, AP India. His research fields are in Artificial Intelligence, Wireless Networks, Cryptography, Algorithms, Data Security.

Volume 6 Issue 12, December 2017

www.ijsr.net 